\title{
A CASE OF ABDOMINOSCROTAL HYDROCELE IN ADULT WITH HYDRONEPHROSIS
}

Ravi kant Kumar, Rohit Kumar Jha, Nishith Ekka, Manish Kumar, Shital Malua,
1. Junior Resident. Department of General Surgery, Rajendra Institute of Medical Sciences.Ranchi
2. Senior Resident. Department of General Surgery, Rajendra Institute of Medical Sciences. Ranchi
3. Senior Resident. Department of General Surgery, Rajendra Institute of Medical Sciences. Ranchi
4. Junior Resident. Department of General Surgery, Rajendra Institute of Medical Sciences. Ranchi
5. Associate Professor. Department of General Surgery, Rajendra Institute of Medical Sciences. Ranchi

\section{CORRESPONDING AUTHOR:}

Dr. Ravikant Kumar,

C/o Dr. S. Malua,

Department of Surgery,

RIMS, Ranchi.

E-mail: dial4ravi@gmail.com

ABSTRACT:_Abdominoscrotal hydrocele is a rare clinical condition. Very few cases have been reported in literature. This case report is of a 35 years old adult male patient presenting as right sided abdominoscrotal hydrocele and its characteristic clinical, imaging and operative findings.

KEY WORDS: Hydrocele, Abdominoscrotal hydrocele.

INTRODUCTION: Abdominoscrotal hydrocele is an unusual clinical condition. Very few cases have been reported in literature, most of which are in children.ASH accounts for about $0.17 \%$ of all cases of hydrocele ${ }^{1}$.ASH consists of huge dumbbell-shaped hydrocele in which scrotal and abdominal components are communicating through deep ring.

CASE REPORT: A 35 years old previously healthy male presented with right sided scrotal swelling since 2 years and abdominal swellings since 6 months. The swelling was associated with dull aching pain in lower abdomen in lower abdomen and right inguinoscrotal region. He had no history of fever and trauma. On clinical examination, a well defined lump noted in lower abdomen extending to scrotum on right side through right inguinal canal. Penis was deviated towards left. The swelling was mildly tender, tense, cystic, and revealed cross fluctuation between scrotal and inguinal components. The right testis was not palpable separately from swelling.

Figure 1: abdominoscrotal swelling in the right lower abdomen extending in to right groin and scrotum

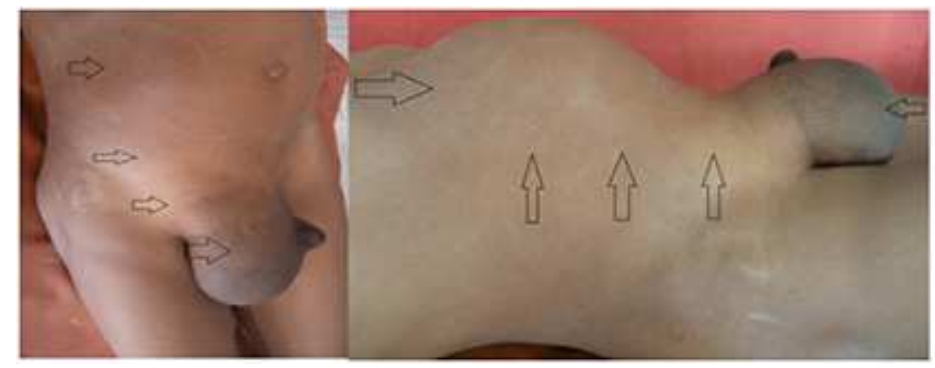


Ultrasonography of abdomen and right inguinoscotal region revealed intra-abdominal cyst communicating with scrotum and right sided hydronephrosis.CT scan of abdomen was consistent with finding of USG except it also revealed mild left sided hydronephrosis.

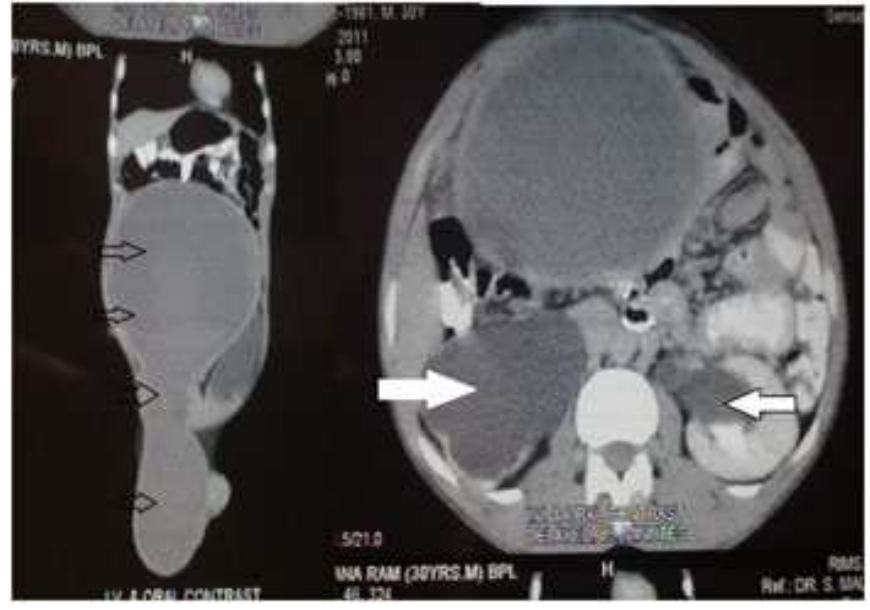

Figure 2: Contrast enhanced CT Scan of abdomen showing large cyst communicating between scrotal and abdominal cavities in coronal view(indicated by black arrow)and bilateral hydronephrosis(gross on right side and mild on left side)in sagittal view indicated by white arrows)

A lower midline laparotomy was performed along with separate right inguino-scrotal incision, complete excision of cyst along with right testes was performed as testes was found atrophic. The postoperative recovery was uneventful. Histopathological examination of cyst wall revealed fibrous tissue and collagen bundles. In testes normal seminiferous tubules with maturation arrest and normal glandular elements was found. There was no any evidence of malignancy. Examination of aspirated fluid from was grossly, microscopically and cytologically consistent with hydrocele fluid.

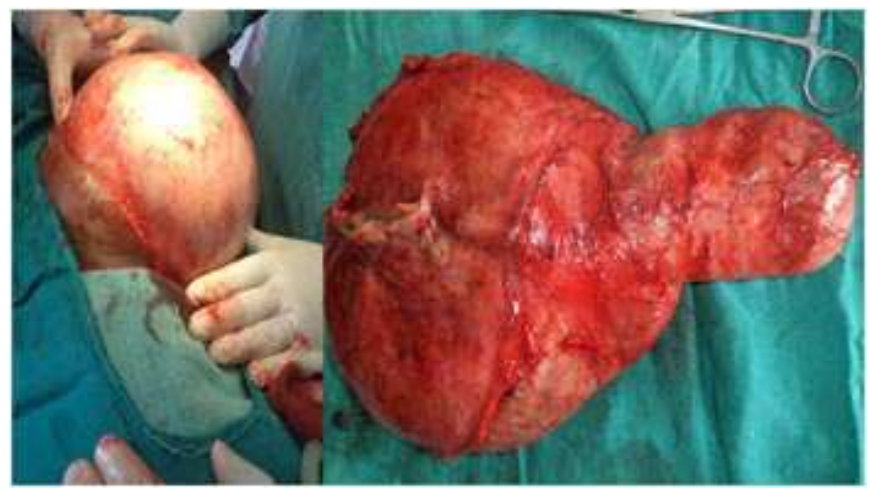

Figure 3: operative finding large abdominoscrotal hydrocele and excised specimen

DISCUSSION: Abdominoscrotal hydrocele consists of large abdominal component that communicates with scrotal component in shape of a dumbbell through deep ring. Dupuytren first described the condition in 1834 as 'hydrocele enbissac' (collections of fluid in the tunica vaginalis, which extends from the scrotum to the abdominal cavity) ${ }^{2}$. The proposed theory of 
ASH is related to partial obliteration of the processus vaginalis, which serves as a 1-way valve to 'pump up' the scrotal portion of the hydrocele with intraperitoneal fluid during episodes of high intra-abdominal pressure. At times, when the intrascrotal pressure exceeds the intra-abdominal pressure, the proximal (intra-abdominal) portion of the hydrocele expands, thus the ASH becomes a dumbbell configuration with central constriction at the inguinalring. ${ }^{3}$

The diagnosis can be made clinically by bimanual palpation of a dumbbell shaped, cystic swelling with cross fluctuation. The first investigation is USG and usually diagnostic. Ultrasound revealed cystic fluid collections extending from the abdomen to the scrotum through an inguinal ring. CT or MRI would help to know the extents of the ASH. MRI is useful for excluding other conditions such as testicular tumour, vas deferens cyst, or lymphangioma. Complications of ASH are hydronephrosis, or unilateral leg oedema as a result of compression on the ureter and iliac vein, respectively.4, 5 imaging would be useful to rule out complications. Testicular changes and interference with spermatogenesis has been reported. 6 The excision of sac is recommended treatment for ASH. Total excision of sac is necessary to prevent reccurence.

CONCLUSION: Abdominoscrotal hydrocele in adults is a very rare clinical entity. This can be diagnosed clinically with added help of imaging techniques. Complete excision is the treatment of choice.

\section{REFRENCES:}

1. Broadman HR, Broadman LEB, Broadman RF, Etiology of abdominoscrotal hydrocele. Urology 1997;10: 564-5.

2. Dupuytren G. Leconsorales de cliniquechirugicale. Balliere. 1834;4:444.

3. DD Rasalkar, WCW Chu, B Mudalgi, BK Paunipagar, J HK CollRadiol. 2009;12:76-78

4. Krasna IH, Solomon M, Mezrich R. Unilateral leg edema caused byabdominoscrotal hydrocele: elegant diagnosis by MRI. J PediatrSurg. 1992; 27:1349-51.

5. Klin B, Efrati Y, Mor A. Vinograd I. Unilateral hydroureteronephorosis caused by abdominoscrotal hydrocele. J Urol. 1992; 148: 384-6.

6. Dandapat MC, Padhi NC, Patra AP. Effect of hydrocele on testes and spermatogenesis. Br J Surg. 1990; 77:1293-4. 